\title{
Bidirectional shooting method for extreme nonlinear optics
}

\author{
A. Hofstrand, ${ }^{1,2}$ P. Jakobsen, ${ }^{3,2}$ and J. V. Moloney ${ }^{2,1}$ \\ ${ }^{1}$ Program in Applied Mathematics, University of Arizona, Tucson, Arizona 85721, USA \\ ${ }^{2}$ College of Optical Sciences, University of Arizona, Tucson, Arizona 85721, USA \\ ${ }^{3}$ Department of Mathematics and Statistics, University of Tromso, 9037 Tromso, Norway
}

(Received 12 September 2019; revised manuscript received 16 October 2019; published 11 November 2019)

\begin{abstract}
In this paper we introduce a pseudospectral shooting method to model intense, ultrashort optical pulses incident on remote targets. The method naturally extends the unidirectional pulse propagation equation to include backward propagating radiation due to either nonlinear coupling with the "forward" field or reflections generated at material boundaries. The general applicability of the method is highlighted with several examples, including a full simulation of a pulse propagating through air across an ultrathin gold film coated on fused silica.
\end{abstract}

DOI: 10.1103/PhysRevA.100.053818

\section{INTRODUCTION}

In the field of extreme nonlinear optics, interactions of ultrashort pulses (USPs) with materials are strongly nonperturbative and generate spectral responses spanning many octaves. In this regime capturing the correct physics becomes extremely challenging. New intense USP laser sources are rapidly coming online, opening new scientific opportunities at longer mid-wavelength infrared [1] and long-wavelength infrared [2] wavelengths. For atmospheric applications, the impact of turbulent scattering is reduced, and as the critical power scales with wavelength squared, it is possible to transport hundreds of gigawatts to terawatts in a single nonlinear filament. In particular, the very large powers available within individual filaments, together with the relatively flat dispersion landscape within atmospheric transparency windows at longer wavelengths, make it possible to efficiently generate significant powers in remote higher harmonics or even $\mathrm{THz}$ wavelengths. When modeling long-range atmospheric propagation, phase matching between the fundamental and the generated harmonics is extremely sensitive and cumulative, requiring an accurate, ultrabroadband representation of relevant responses. Although envelope models have been the method of choice for simulating filaments [3], they fail to capture this situation. Thus, one must resort to physical models that resolve the underlying optical carrier wave and, for efficient wavelength conversion, capture both ultrabroadband linear and nonlinear spectral responses. Moreover, in such extremes, the potential for significant backward scattered or self-generated fields may appear [4]. For instance, experimentalists interested in remote sensing of the atmosphere have detected enhanced, ultrabroadband back-scattered radiation due to the filamentation process of unidirectionally sourced intense USPs [5,6]. The generation of high-gain backward lasing in air via a remote USP pump is also being explored as a means to monitor the atmosphere [7].

The unidirectional pulse propagation equation (UPPE) $[8,9]$ has emerged as the computational tool of choice as a nonlinear pseudospectral solver capable of capturing extreme broadband generation. The strength of the UPPE is that it solves the linear propagator exactly and is able to accurately resolve complicated nonlinear dynamics which accumulate over a much longer propagation scale. Another strength of the UPPE is the ability to resolve nonparaxial propagation dynamics [10]. Recent papers have explored the validity of the unidirectional approximation in the $\mathrm{THz}$ regime [11] and for tightly focused pulses $[12,13]$. Although the UPPE can be generalized for particular waveguiding structures using eigenmode expansions [14], the method cannot currently account for nonlinearly induced back-scattering or sharp gradients in the index of refraction along the direction of propagation. These limitations are obvious as the UPPE is derived explicitly assuming the backward and transversely propagating modes are weak, which is valid for many problems of physical interest.

We now pose the problem of how to realistically model an USP's interaction with a remote scattering boundary. One possible approach is to couple the output of the UPPE solver with a full Maxwell solver once near the remote target. Very few full Maxwell solvers currently exist that can resolve potentially few-cycle, intense pulse-scattering interactions in nonlinear media. The finite difference time domain (FDTD) solver [15,16] represents the current state of the art for studying full-field interactions in linear and possibly nonlinear media. However, this scheme is extremely restrictive, being limited to simulating small-scale objects in three dimensions due to the Courant-Friedrichs-Lewy stability condition and is plagued by accumulating numerical dispersion errors. As an example of the restrictions inherent in the FDTD, consider a circumstance where it is necessary to resolve a seventh harmonic. To avoid numerical dispersion, this would require a minimum of 20 spatial points at the seventh harmonic's wavelength (and concomitant fine time step), which would then enforce a 140 spatial point resolution of the fundamentala major overkill which blows up computational resources. Modeling nonlinear problems with the FDTD method can also lead to high-frequency instabilities. Another drawback of the FDTD is the lack of a systematic way to incorporate 

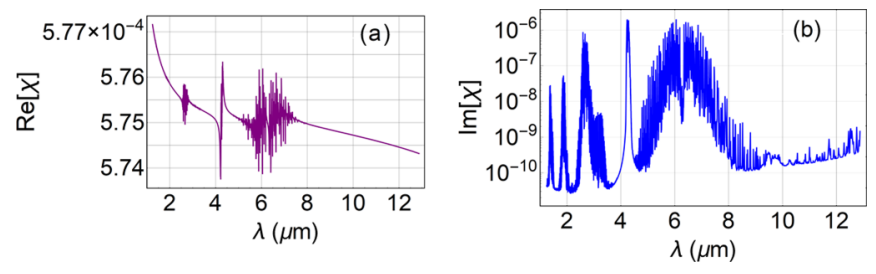

FIG. 1. Dispersion and absorption of infrared light in the atmosphere (HITRAN [17]). (a) Real and (b) imaginary parts of the electric susceptibility.

complicated, ultrabroadband dispersive effects into the simulation. The UPPE currently uses the large HITRAN database [17], shown in Fig. 1, to model these effects, which would be impossible to implement in a time-domain solver for femtosecond-duration pulses.

A more accurate and streamlined approach to simulating such pulses would be to somehow include back-scattering modes into the UPPE formalism. These modes could be generated from purely forward-propagating modes in two ways: A weak nonlinear coupling between the modes and/or a nonzero gradient in the index of refraction along the direction of pulse propagation. Both of these scenarios are addressed in this article. In Sec. II we derive the bidirectional pulse propagation equation (BPPE) in complete generality with no simplifying assumptions (i.e., no paraxial, unidirectional, slowly varying-envelope approximations, etc.). We also present a shooting method which can be used to solve problems of broadband nonlinear pulse propagation across stratified boundary layers. Section III consists of several examples of the BPPE shooting method, including (1) a converged example of nonnormal incidence, $(2)$ a full $(2+1)$ simulation of a realistic pulse propagating across an ultrathin gold film, and (3) the formation of a near-shock front in the carrier wave of an USP traveling across an air-diamond interface. We also outline some results on the convergence of the BPPE iteration scheme and fill in some details on how our simulations were executed in the Appendix.

\section{BPPE SHOOTING METHOD}

To begin this section we sketch a derivation of the scalar BPPE. We first consider the case of propagation without material boundaries, i.e., backward-propagating radiation is solely due to nonlinear coupling with the "forward" field. For simplicity, we assume that the electric field is linearly polarized and the background media are homogeneous, then Maxwell's equations can be cast as a scalar wave equation with a nonlinear source term

$$
\begin{aligned}
\Delta E(\mathbf{x}, t) & -\frac{1}{c^{2}} \partial_{t t}\left[E(\mathbf{x}, t)+\int_{-\infty}^{t} \chi^{(1)}(t-\tau) E(\mathbf{x}, \tau) d \tau\right] \\
& =\frac{1}{c^{2}} \partial_{t t} P_{\mathrm{NL}}[E](\mathbf{x}, t),
\end{aligned}
$$

where $\chi^{(1)}$ is the linear susceptibility and $P_{\mathrm{NL}}$ is the nonlinear part of the polarization response. We let $z$ be the direction of propagation, take the Fourier transform of (1) $\left[(t, x, y) \rightarrow\left(\omega, k_{x}, k_{y}\right)=\left(\omega, \mathbf{k}_{\perp}\right)\right]$, and define the $z$ component of the wave vector as $\beta=+\sqrt{n^{2}(\omega) \omega^{2} / c^{2}-k_{\perp}^{2}}$, where $n(\omega)$ is the material's frequency-dependent index of refraction, to obtain

$$
\partial_{z z} \hat{E}\left(z, \omega, \mathbf{k}_{\perp}\right)+\beta^{2}\left(\omega, k_{\perp}\right) \hat{E}=\frac{-\omega^{2}}{c^{2}} \hat{P}_{\mathrm{NL}}[E]\left(z, \omega, \mathbf{k}_{\perp}\right) .
$$

Equation (2) is essentially a second-order, coupled system of nonlinear ordinary differential equations indexed over the continuous parameters $\omega$ and $\mathbf{k}_{\perp}$. Indeed, from a numerical perspective (2) becomes a finite system via discrete Fourier transforms. The linear version of (2) clearly has the two linearly independent solutions, $A_{+}\left(\omega, \mathbf{k}_{\perp}\right) \exp (i \beta z)$ and $A_{-}\left(\omega, \mathbf{k}_{\perp}\right) \exp (-i \beta z)$. Equation (2) reduces to a first-order system in $z$ if we view the "weak" nonlinear term on the right-hand side of (2) as an inhomogeneity and apply the elementary variation of parameters method [18]. Recall that this entails allowing the coefficient terms in the homogeneous solution to depend on $z$, so we have the expansion

$$
\hat{E}\left(z, \omega, \mathbf{k}_{\perp}\right)=A_{+}\left(z, \omega, \mathbf{k}_{\perp}\right) e^{i \beta z}+A_{-}\left(z, \omega, \mathbf{k}_{\perp}\right) e^{-i \beta z},
$$

and we need to enforce the free condition

$$
\partial_{z} A_{+}\left(z, \omega, \mathbf{k}_{\perp}\right) e^{i \beta z}+\partial_{z} A_{-}\left(z, \omega, \mathbf{k}_{\perp}\right) e^{-i \beta z}=0 .
$$

Substituting (3) into (2) and using (4) gives the scalar BPPE:

$$
\begin{aligned}
& \partial_{z} A_{+}\left(z, \omega, \mathbf{k}_{\perp}\right)=\frac{i \omega^{2}}{2 c^{2} \beta} P_{\mathrm{NL}}\left[A_{+}, A_{-}\right]\left(z, \omega, \mathbf{k}_{\perp}\right) e^{-i \beta z}, \\
& \partial_{z} A_{-}\left(z, \omega, \mathbf{k}_{\perp}\right)=-\frac{i \omega^{2}}{2 c^{2} \beta} P_{\mathrm{NL}}\left[A_{+}, A_{-}\right]\left(z, \omega, \mathbf{k}_{\perp}\right) e^{i \beta z} .
\end{aligned}
$$

Note if we make the unidirectional approximation, which assumes the nonlinear response can be decoupled, system (5) becomes two scalar UPPEs. It should be emphasized that the second-order system (2) is inherently a boundary value problem, whereas the first-order BPPE (5) is cast as an initial value problem. Here we are interested in problems where $A_{+}$is specified at some initial point $z_{0}$, although $A_{-}\left(z_{0}\right)$ is unknown. To solve this problem, we use an iterative method [19], which is based on integrating (4) over $\left[z_{0}, Z\right]$ :

$$
A_{-}(Z)-A_{-}\left(z_{0}\right)=-\int_{z_{0}}^{Z} \partial_{z} A_{+} e^{2 i \beta z} d z \text {. }
$$

If we assume that $A_{-}(Z) \approx 0$ (i.e., there are no material reflections or nonlinear couplings to backward-generated radiation past $Z$ ), we can define the update relation

$$
A_{-}^{(i+1)}\left(z_{0}\right)=\frac{i \omega^{2}}{2 c^{2} \beta} \int_{z_{0}}^{Z} \hat{P}_{\mathrm{NL}}\left[E^{(i)}\right] e^{i \beta z} d z
$$

where we take an initial guess $A_{-}^{(0)}\left(z_{0}\right)=0$, solve system (5), update our guess to $A_{-}^{(1)}\left(z_{0}\right)$ using (7) while keeping $A_{+}\left(z_{0}\right)$ constant, and repeat.

We now present an explicit iterative procedure to extend the BPPE over $z$-dependent stratified boundary layers, as shown in Fig. 2. We refer to our complete scheme as the BPPE shooting method. As will be shown, the method is able to accurately resolve the nonlinear spectral evolution of both the reflected and transmitted pulses. For simplicity, consider a single planar interface located at $z_{1}\left(z_{0}<z_{1}<Z\right)$ which separates two materials (labeled 0 and 1), each having its own dispersion relation, $n_{j}(\omega)$, and Kerr nonlinear index, 


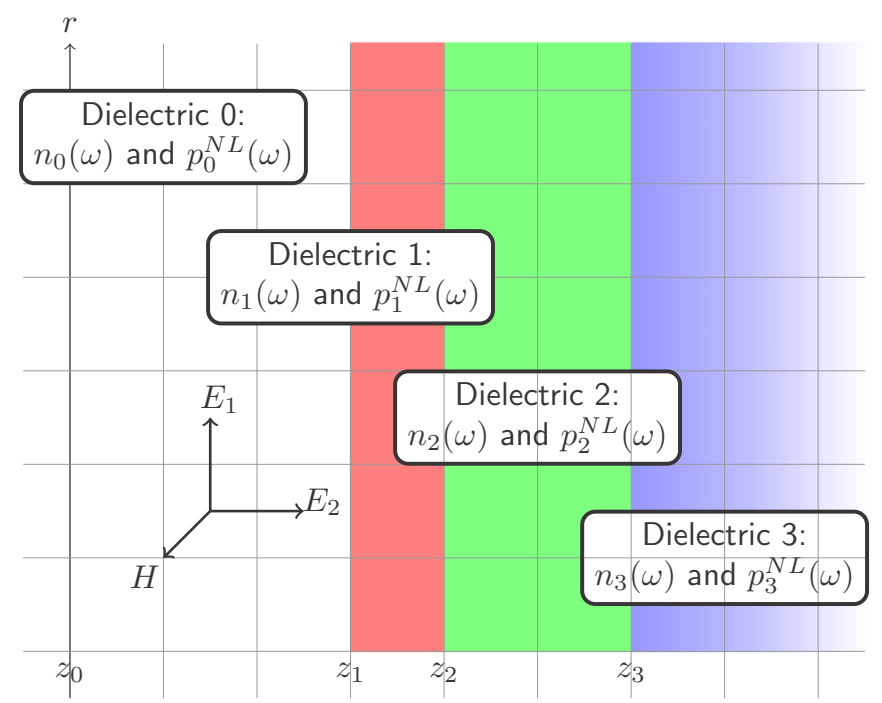

FIG. 2. The BPPE shooting method can capture realistic and complicated linear, $n_{j}(\omega)$, and nonlinear, $p_{j}^{N L}(\omega)$, physics for a bidirectional pulse propagating across stratified layers in $z$.

$n_{2, j}$. Here we consider the $(2+1)$ (space+time) problem and assume the fields are transverse magnetic (TM). A more careful derivation of system (5), based directly on the vector nature of Maxwell's equations, leads to the inclusion of a transverse spectral mode, $Q\left(z, \omega, k_{x}\right)$, into (5) [19]. This mode is driven by a nonlinear response in the direction of propagation, $p_{z}$, which for near-bidirectional propagation is extremely weak. We include this mode for completeness. It is now a straightforward matter to enforce the field boundary conditions across the interface at $z_{1}$ in terms of the spectral amplitudes. Let $z_{1}^{-}$and $z_{1}^{+}$denote just to the left and right of the boundary, respectively. Ensuring the continuity of the tangential components of $\mathbf{E}$ and $\mathbf{B}$ as well as the normal component of $\mathbf{D}$ at $z_{1}$ leads to

$$
\mathbf{M}_{\mathbf{0}} \mathbf{A}\left(z_{1}^{-}\right)+\hat{p}_{z}\left(z_{1}^{-}\right) \hat{\mathbf{z}}=\mathbf{M}_{\mathbf{1}} \mathbf{A}\left(z_{1}^{+}\right)+\hat{p}_{z}\left(z_{1}^{+}\right) \hat{\mathbf{z}},
$$

where for $j=0,1$,

$$
\mathbf{M}_{\mathbf{j}}=\left[\begin{array}{ccc}
e^{i \beta_{j} z_{1}} & e^{-i \beta_{j} z_{1}} & 0 \\
g_{j} e^{i \beta_{j} z_{1}} & -g_{j} e^{-i \beta_{j} z_{1}} & -k_{x} \\
-h_{j} e^{i \beta_{j} z_{1}} & h_{j} e^{-i \beta_{j} z_{1}} & n_{j}^{2}
\end{array}\right]
$$

and

$$
\begin{gathered}
\mathbf{A}(z)=\left[\begin{array}{c}
A_{+}\left(z, \omega, k_{x}\right) \\
A_{-}\left(z, \omega, k_{x}\right) \\
Q\left(z, \omega, k_{x}\right)
\end{array}\right], \\
g_{j}\left(\omega, k_{x}\right)=\beta_{j}\left(\omega, k_{x}\right)+k_{x}^{2} / \beta_{j}\left(\omega, k_{x}\right), \\
h_{j}\left(\omega, k_{x}\right)=n_{j}^{2}(\omega) k_{x} / \beta_{j}\left(\omega, k_{x}\right) .
\end{gathered}
$$

To leading order, expressions (8)-(11) yield a system of linear equations which must be solved at each spectral grid point across the boundary at $z_{1}$. System (8)-(11) can now be included with the iterative method outlined in the preceding paragraph to model the nonlinear propagation of an USP traveling across a material boundary. We give the explicit steps of the BPPE shooting method as well as some details on convergence in the Appendix. Now we turn to a numerical implementation of the method and display converged results.

\section{PHYSICAL EXAMPLES}

In this section, we highlight the applicability of the BPPE shooting method with several examples. First, we consider the linear propagation of a pulse with nonnormal incidence across an air-diamond interface. We include this simple linear example here to validate convergence of the fundamental scheme. The results of this simulation are shown in Fig. 3.

We use the Sellmeier equations for air and bulk diamond given in Refs. [20,21] and simulate a pulse interacting with the boundary at a $20^{\circ}$ angle of incidence. Remarkably, the solution converges to the correct, angle-dependent Fresnel law of reflection and transmission with good accuracy after only five iterations of the BPPE shooting method. In the simple case of linear propagation, an explicit angle-and-frequencydependent convergence requirement for our iteration scheme can be calculated (see the Appendix). As the BPPE shooting method is based on bidirectional propagation along the $z$ axis, it is expected (and confirmed in the Appendix) that for near-perpendicular angles the BPPE shooting method should diverge. It is clear that both the UPPE and BPPE (5) fail when $\beta \approx 0$. Fig. 3(d) shows the power spectrum of the initial pulse along with the line $\beta=0$. Spectral components sufficiently close to $\beta=0$ need to be filtered out since they will eventually diverge. This is problematic only when the pulse spectrum lies on top of $\beta=0$ and as seen in Fig. 3(d) is not an issue here. We now turn to nontrivial nonlinear BPPE shooting method simulations.
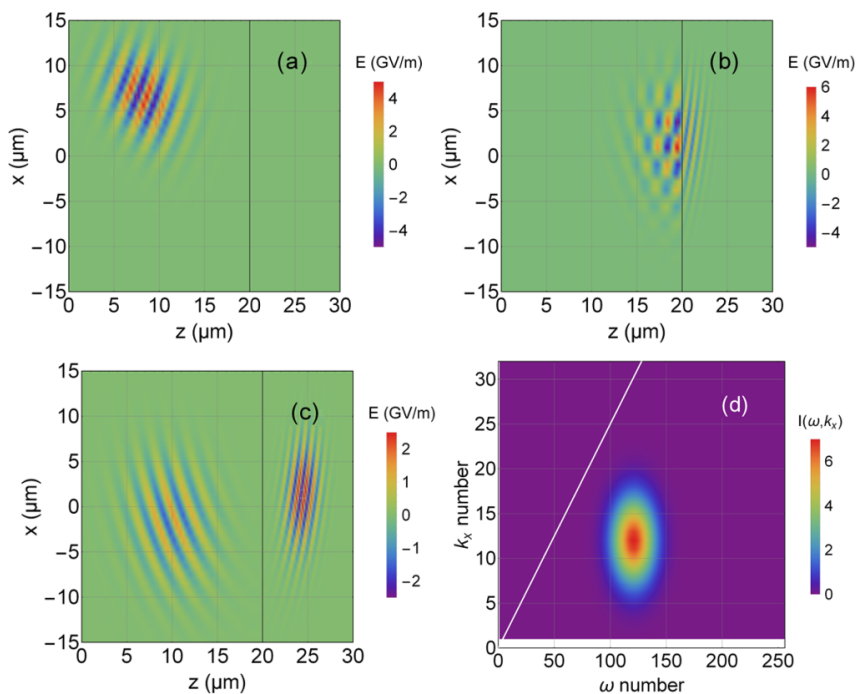

FIG. 3. BPPE shooting method simulation (after five iterations) of a $20 \mathrm{fs}$ pulse ( $e^{-2}$ radius) with a central wavelength of $2 \mu \mathrm{m}$ and an initial wavelength-scale beam waist of $6 \mu \mathrm{m}$ ( $e^{-2}$ radius) propagating across an air-diamond interface at $20^{\circ}$. (a), (b), and (c) The diffracting real field entering the domain from the left, hitting the boundary and the resulting transmitted and reflected pulses, respectively. (d) The spectral density of the initial pulse with the light line, $\beta=0$, plotted for reference. 

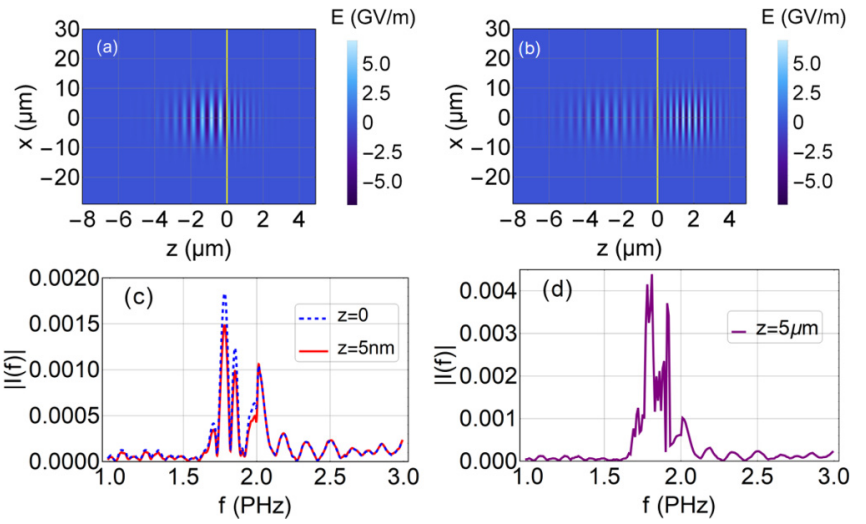

FIG. 4. $(2+1)$ nonlinear BPPE shooting method simulation of a short 10fs pulse with a central wavelength of $\lambda_{0}=0.5 \mu \mathrm{m}$ $(\sim 0.6 \mathrm{PHz})$ and beam waist of $10 \mu \mathrm{m}$ after 10 iterations propagating across $5 \mathrm{~nm}$ gold film. The initial peak intensity of the beam is $4.0 \mathrm{TW} / \mathrm{cm}^{2}$. The field, $E_{x}(z, x, t)$, is shown at times (a) $25.50 \mathrm{fs}$ and (b) $35.28 \mathrm{fs}$. (c) The on-axis spectral intensity of the generated third harmonic just before the pulse hits the gold film (blue) and after propagating through the film (red); (d) the spectral intensity of the third harmonic at the end of propagation in silica.

Next we simulate the nonlinear propagation of an intense USP traveling through air at normal incidence into a block of fused silica coated with a $5 \mathrm{~nm}$ film of gold. The optical response of ultrathin metallic films is also an active area of applied research [22]. For simplicity, only the instantaneous Kerr effect of air and silica are included in the nonlinear polarization of our simulation $\left(n_{2}=5.36 \times 10^{-23} \mathrm{~m}^{2} \mathrm{~W}^{-1}\right.$ and $n_{2}=3.00 \times 10^{-20} \mathrm{~m}^{2} \mathrm{~W}^{-1}$, respectively). More complicated nonlinear responses such as stimulated Raman effects and plasma generation could be included in the BPPE iteration scheme in the same way they are implemented in UPPE solvers [9]. The dispersion landscapes for air and silica are modeled using appropriate Sellmeier equations while the dispersive response of the ultrathin gold film is modeled with interpolated experimental data given in Ref. [23] (data shown in the Appendix). As stated before, the streamlined implementation of dispersive data is a major advantage that both the UPPE and BPPE have over time-domain solvers such as the FDTD. The result of our simulation is shown in Fig. 4. As the nonlinear response is weak in both air and silica over the propagation distances considered, the macroscopic dynamics are essentially linear, although the bottom row of Fig. 4 clearly shows the generation of a third harmonic and its evolution in each medium. Figure 4 shows that the BPPE method with interface quickly converges to the expected near-Fresnel phase and amplitude relations between the incident, reflected, and transmitted pulses with detailed, broadband physical responses included. In application, the initial USP input into the BPPE method could be the output of a kilometer-scale UPPE simulation with a complicated broadened spectrum.

Finally, as a check on the robustness of the BPPE shooting method, we consider the $(2+1)$ propagation of a pulse across a single air-diamond interface with an artificially inflated nonlinear index so that the nonlinear response manifests itself over the short propagation distance considered. We find that even in this rather extreme case the BPPE iteration scheme
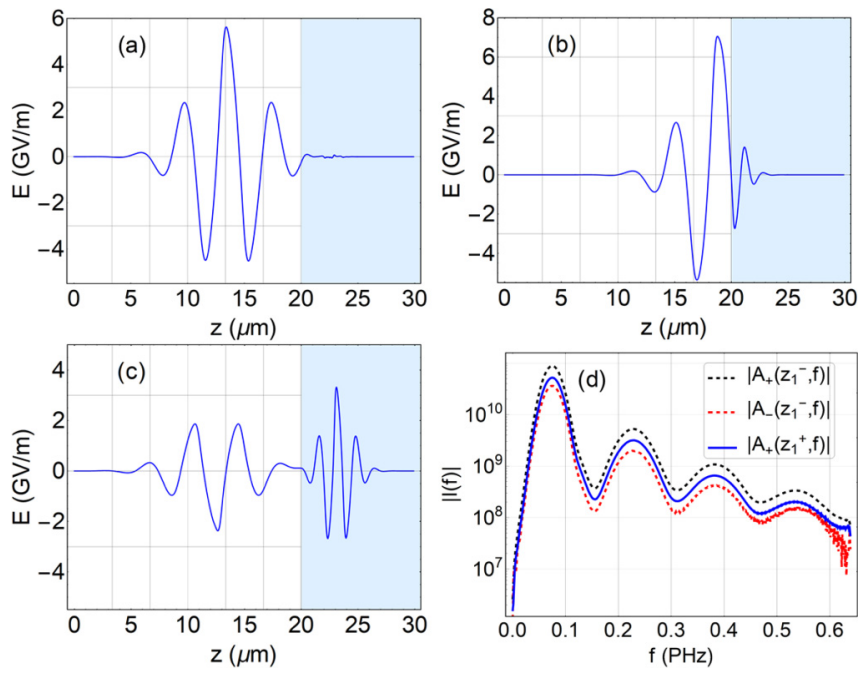

FIG. 5. On-axis profile of a $(2+1)$ nonlinear BPPE simulation of a $20 \mathrm{fs}$ pulse with initial peak intensity of $4.0 \mathrm{TW} / \mathrm{cm}^{2}$ centered at $\lambda_{0}=4 \mu \mathrm{m}$ propagated across an air-diamond interface with an artificially inflated nonlinear response in air after 20 iterations at various times (a)-(c). (d) The on-axis spectral intensity of the incident, reflected, and transmitted pulses.

converges. Figure 5 shows the pulse's carrier wave forming a near-shock front ("shark-fin"), due to the weak dispersion and losses of air and diamond around $4 \mu \mathrm{m}$. This behavior was first observed in intense, few-cycle MWIR pulses using the UPPE [24]. The carrier-wave steepening in the incident, reflected, and transmitted pulses generates a significant and broad spectral response of odd higher harmonics, which are accurately resolved in the lower right plot in Fig. 5.

\section{CONCLUSION}

We have shown that the UPPE can be extended using the BPPE method to accurately simulate the bidirectional, nonlinear dynamics of an USP propagating across sharp physical interfaces. In practice, a pulse could be propagated over kilometer-scale distances using the UPPE, resulting in significant pulse distortion and spectral broadening, and then seamlessly connected with the BPPE solver to propagate the pulse across boundary layers or to produce localized, nonlinearly generated back-scattered radiation. The output of the BPPE simulation could then be inputed back into decoupled "left" and "right" UPPE solvers, continuing multiple pulse trajectories. In this manner, the BPPE shooting method offers a streamlined approach to accurately model ultrabroadband, near-bidirectional USP physics.

\section{ACKNOWLEDGMENT}

This material is based on work supported by the Air Force Office of Scientific Research under Grant No. FA9550-19-1-0032. 


\section{APPENDIX: BPPE SHOOTING METHOD: PROCEDURE AND CONVERGENCE}

We first state the explicit steps of the BPPE shooting method discussed in Sec. II. For simplicity of notation we consider the $(1+1) \mathrm{TM}$ problem so that $k_{x}, Q \equiv 0$, and to leading order (8)-(11) is a $2 \times 2$ linear system defined at each spectral grid point. The BPPE procedure for a single interface at $z_{1}$ is summarized in the following steps:

1. Set $A_{+}\left(z_{0}, \omega\right)$ to a given forward-propagating field and the initial backward-propagating complex amplitude $A_{-}^{(0)}\left(z_{0}, \omega\right)$ to zero. Solve the BPPE (5) through material 0 to $z_{1}$.

2. Solve system (8)-(11) to obtain $A_{+}\left(z_{1}^{+}, \omega\right)$ and $A_{-}^{(0)}\left(z_{1}^{+}, \omega\right)$.

3. Solve the BPPE given the initial values from step 2 through material 1 to $Z$.

4. To update iteration $A_{-}^{(0)}\left(z_{0}, \omega\right)$, define

$$
A_{-}^{(1)}\left(z_{0}, \omega\right)=\widetilde{A}_{-}^{(0)}\left(z_{1}^{-}, \omega\right)+\int_{z_{0}}^{z_{1}} \partial_{z} A_{+} e^{2 i \beta_{0} z} d z
$$

where

$$
\widetilde{A}_{-}^{(0)}\left(z_{1}^{+}, \omega\right)=\int_{z_{1}}^{Z} \partial_{z} A_{+} e^{2 i \beta_{1} z} d z
$$

and $\widetilde{A}_{-}^{(0)}\left(z_{1}^{-}, \omega\right)$ is obtained from the equation above and using the boundary conditions (8)-(11) again at $z_{1}$.

5. Update the iteration and repeat:

$$
A_{-}^{(0)}\left(z_{0}, \omega\right) \rightarrow A_{-}^{(1)}\left(z_{0}, \omega\right) \text {. }
$$

We now give some conditional statements for linear convergence for the above BPPE method when including boundary interfaces. For the simplest case of linear, one-dimensional propagation for a single frequency, $\omega_{0}$, the entire BPPE shooting method outlined above can be compactly expressed as

$$
\left[\begin{array}{l}
A_{+}^{(k+1)}\left(z_{0}\right) \\
A_{-}^{(k+1)}\left(z_{0}\right)
\end{array}\right]=\mathcal{M}\left[\begin{array}{c}
A_{+}^{(k)}\left(z_{0}\right) \\
A_{-}^{(k)}\left(z_{0}\right)
\end{array}\right]+\left[\begin{array}{c}
A_{+}^{(k)}\left(z_{0}\right) \\
0
\end{array}\right],
$$

where $\mathcal{M}=\boldsymbol{\Gamma}_{\mathbf{1}} \mathbf{M}_{\mathbf{0}}{ }^{-1} \mathbf{M}_{\mathbf{1}} \boldsymbol{\Gamma}_{\mathbf{0}} \mathbf{M}_{\mathbf{1}}{ }^{-1} \mathbf{M}_{\mathbf{0}}, k$ is the iteration number, and $j=0,1$ is the material label

$$
\begin{gathered}
\mathbf{M}_{\mathbf{j}}=\left[\begin{array}{cc}
e^{\mathrm{in}{ }_{j}\left(\omega_{0}\right) \omega_{0} z_{1} / c} & e^{-\mathrm{in}_{j}\left(\omega_{0}\right) \omega_{0} z_{1} / c} \\
n_{j}\left(\omega_{0}\right) e^{\mathrm{in}{ }_{j}\left(\omega_{0}\right) \omega_{0} z_{1} / c} & -n_{j}\left(\omega_{0}\right) e^{-\mathrm{in}_{j}\left(\omega_{0}\right) \omega_{0} z_{1} / c}
\end{array}\right], \\
\boldsymbol{\Gamma}_{j}=\left[\begin{array}{cc}
\delta_{j 0} & 0 \\
0 & \delta_{j 1}
\end{array}\right] .
\end{gathered}
$$

Using (A1) and (A2), it can be explicitly shown that the method converges to the expected Fresnel law of reflection and transmission if the index step satisfies

$$
\mathcal{C}\left(\omega_{0} ; n_{0}, n_{1}\right)=\left|\frac{\left(n_{1}\left(\omega_{0}\right)-n_{0}\left(\omega_{0}\right)\right)^{2}}{4 n_{0}\left(\omega_{0}\right) n_{1}\left(\omega_{0}\right)}\right|<1 .
$$

For instance, if $n_{0}\left(\omega_{0}\right)=1$, convergence is achieved under the condition $n_{1}\left(\omega_{0}\right)<3+2 \sqrt{2} \approx 5.83$, which is clearly fulfilled for many problems of physical interest. The linear convergence condition for a single interface can be generalized in $(2+1)$ for a pulsed source to the expression given in (A4), which reduces to (A3) when $k_{x}=0$. For instance, the linear simulation shown in Fig. 3 contains only spectral components, so that condition (A4) holds. Since the problematic components play a minimal role in the overall dynamics it is straightforward to filter them out:

$$
\mathcal{C}\left(\omega, k_{x} ; n_{0}, n_{1}, \beta_{0}, \beta_{1}\right)=\frac{1}{4}\left|\frac{\left[-n_{0}^{2} n_{1}^{2} \omega^{2}+c^{2}\left(k_{x}^{2} n_{1}^{2}+n_{0}^{2} \beta_{0} \beta_{1}\right)\right]\left[-n_{0}^{2} n_{1}^{2} \omega^{2}+c^{2}\left(k_{x}^{2} n_{0}^{2}+n_{1}^{2} \beta_{0} \beta_{1}\right)\right]}{n_{0}^{2} n_{1}^{2}\left(c^{2} k_{x}^{2}-n_{0}^{2} \omega^{2}\right)\left(c^{2} k_{x}^{2}-n_{1}^{2} \omega^{2}\right)}\right|<1 .
$$

The preceding analysis can be generalized further to include multiple stratified boundary layers. This leads to an analytic convergence condition for the linear gold film simulation outlined in Sec. III. For reference, the dispersive data used in that simulation are shown in Fig. 6. The full convergence condition, $\mathcal{C}\left(f_{c}, k_{x, c}, w ; n_{0}, n_{1}, n_{2}\right)$, depends on the width of the gold film $(w)$, the central frequency $\left(f_{c}\right)$ and transverse wave number $\left(k_{x, c}\right)$ components of the pulse, as well as the corresponding refractive indices in all three materials. As the derived expression is long and messy, we do not include it here. Instead, we plot the obtained convergence condition versus frequency and wave number in Fig. 7 for the particular model parameters used for the gold film simulation in Sec. III. The range in frequencies and wave numbers in Fig. 7(a) matches our numerical grid. As long as our derived condition, $\mathcal{C}$, is strictly less than one, the linear BPPE scheme will converge. We have also verified that the linear BPPE method diverges when including spectral components outside of the window $\mathcal{C}<1$. The two diagonal lines clearly seen in Fig. 7(b) match the light curves for air and silica, across which propagating waves become evanescent $\left[k_{x}=n_{j}(\omega) \omega / c\right]$. Clearly, both the UPPE and BPPE diverge here as the evolution equations become unbounded [system (5)]. The divergence of the scalar UPPE and BPPE along the light curves becomes an issue only when considering pulses with high angles of incidence (with respect to $z$-dependent boundaries) and for extreme, subwavelength nonlinear focusing.

The white vertical line in Fig. 7(a) marks where the derived convergence condition, $\mathcal{C}$, is equal to one. Frequencies higher than approximately $4 \mathrm{PHz}$ in our example cause the linear BPPE iteration scheme to diverge. One can easily filter out these problematic frequencies, leaving only the spectral components which are primarily responsible for the pulse's propagation dynamics (third, fifth, etc., harmonics). 


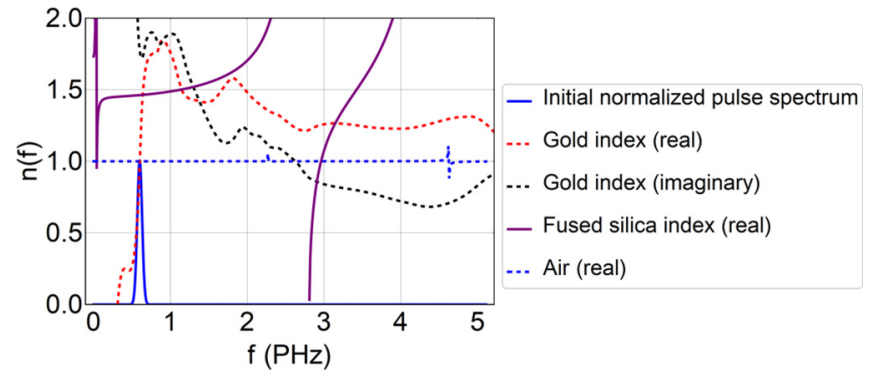

FIG. 6. The linear dispersion data and initial pulse used in the BPPE shooting method simulation shown in Fig. 4.

This was done when simulating the pulse shown in Fig. 4. It should be noted that the strong absorption in the gold film leads to a more restrictive frequency window (and very thin film width) for the BPPE method to converge. This restriction is relaxed when considering stratified dielectric layers.
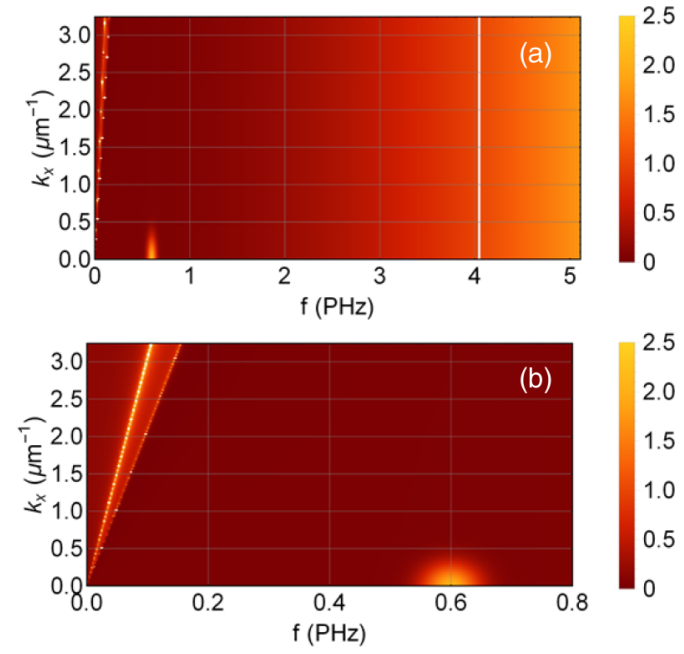

FIG. 7. The linear BPPE convergence condition, $\mathcal{C}$, and scaled initial pulse spectrum used in the gold film example in Sec. III. Convergence is guaranteed if $\mathcal{C}<1$. The domain of panel (a) is the used BPPE computational boundary, and panel (b) is simply panel (a) zoomed in around the initial pulse.
[1] A. V. Mitrofanov, A. A. Voronin, D. A. Sidorov-Biryukov, A. Pugzlys, E. A. Stepanov, G. Andriukaitis, T. Flory, S. Alisauskas, A. B. Fedotov, A. Baltuska, and A. M. Zheltikov, Sci. Rep. 5, 8368 (2015).

[2] D. J. Haberberger, S. Tochitsky, and C. Joshi, Opt. Express 18, 17865 (2010).

[3] A. Couairon and A. Mysyrowicz, Phys. Rep. 441, 47 (2007).

[4] S. Mitryukovskiy, Y. Liu, P. J. Ding, A. Houard, and A. Mysyrowicz, Opt. Express 22, 12750 (2014).

[5] J. Kasparian, M. Rodriguez, G. Mejean, J. Yu, E. Salmon, H. Wille, R. Bourayou, S. Frey, Y. B. Andre, A. Mysyrowicz, R. Sauerbrey, J. P. Wolf, and L. Woste, Science 301, 61 (2003).

[6] Q. Luo, H. A. Xu, S. A. Hosseini, J. F. Daigle, F. Théberge, M. Sharifi, and S. L. Chin, Appl. Phys. B: Lasers Opt. 82, 105 (2005).

[7] A. Dogariu, J. B. Michael, M. O. Scully, and R. B. Miles, Science 331, 442 (2011).

[8] M. Kolesik and J. V. Moloney, Phys. Rev. Lett. 89, 283902 (2002).

[9] M. Kolesik and J. V. Moloney, Phys. Rev. E 70, 036604 (2004).

[10] R. Penjweini, M. Weber, M. Sondermann, R. W. Boyd, and G. Leuchs, Optica 6, 878 (2019).

[11] J. Déchard, A. Nguyen, P. González de Alaiza Martínez, I. Thiele, S. Skupin, and L. Bergé, J. Phys. Commun. 1, 055009 (2017).
[12] M. Kolesik, P. Jakobsen, and J. V. Moloney, Phys. Rev. A 86, 035801 (2012).

[13] P. Kinsler, J. Opt. Soc. Am. B 24, 2363 (2007).

[14] J. Andreasen and M. Kolesik, Phys. Rev. E 86, 036706 (2012).

[15] S. C. Hagness and A. Taflove, Computational Electrodynamics: The Finite-Difference Time-Domain Method, 3rd ed. (Artech House, Boston/London, 2005).

[16] A. Taflove, S. G. Johnson, and A. Oskooi, Advances in FDTD Computational Electrodynamics (Artech House, Boston/London, 2013).

[17] I. E. Gordon, L. S. Rothman, C. Hill et al., J. Quant. Spectrosc. Radiat. Transfer 203, 3 (2017).

[18] K. Glasner, M. Kolesik, J. V. Moloney, and A. C. Newell, Int. J. Opt. 2012, 868274 (2011).

[19] P. Jakobsen, Phys. Scr. 89, 095502 (2014).

[20] P. E. Ciddor, Appl. Opt. 35, 1566 (1996).

[21] H. R. Phillip and E. A. Taft, Phys. Rev. 136, A1445 (1964).

[22] A. Kossoy, V. Merk, D. Simakov, K. Leosson, S. Kena-Cohen, and S. A. Maier, Adv. Opt. Mater. 3, 71 (2015).

[23] H. J. Hagemann, W. Gudat, and C. Kunz, J. Opt. Soc. Am. 65, 742 (1975).

[24] P. Panagiotopoulos, P. Whalen, M. Kolesik, and J. V. Moloney, J. Opt. Soc. Am. B 32, 1718 (2015). 